\title{
Optimized Detection Limits in FIB-SIMS by Using Reactive Gas Flooding and High Performance Mass Spectrometers
}

\author{
T. Wirtz, D. Dowsett, P. Philipp
}

Department "Science and Analysis of Materials" (SAM), Centre de Recherche Public - Gabriel Lippmann, 41 rue du Brill, L-4422 Belvaux, Luxembourg

FIB-based instruments play an ever more important role in materials science and also in life science. While such FIB-based instrumentation is an ideal tool for high resolution imaging (2D and 3D imaging) and nanofabrication (nanomaching, nanodeposition, specimen preparation), its analysis capability is currently limited. By contrast, Secondary Ion Mass Spectrometry (SIMS) is an extremely powerful technique for analyzing surfaces owing in particular to its excellent sensitivity, high dynamic range, very high mass resolution and ability to differentiate between isotopes. Adding SIMS capability to FIB instruments offers not just the prospect of obtaining SIMS information limited only by the size of the probe-sample interaction $(\sim 10 \mathrm{~nm})$ but also enables a direct correlation of such SIMS images with high resolution secondary electron images of the same zone taken at the same time.

Past attempts of performing SIMS on FIB instruments were rather unsuccessful due to unattractive detection limits, which were due to (i) low ionization yields of sputtered particles, (ii) extraction optics with limited extraction and collection efficiency of secondary ions and (iii) mass spectrometers having low duty cycles and/or low transmission. In order to overcome these limitations, we have investigated the use of reactive gas flooding during FIB-SIMS and we have developed compact high-performance magnetic sector mass spectrometers with dedicated high-efficiency extraction optics.

In order to reach good detection limits when probing very small voxels in imaging applications, the ionization probability of the sputtered atoms and molecules needs to be maximized. When using typical ion species used in FIB-based instrumentation such as Ga or noble gases, the intrinsic yields are low compared to the ones found in conventional SIMS. However, the yields may be drastically increased by using reactive gas flooding during analysis, namely $\mathrm{O}_{2}$ flooding for positive secondary ions and Cs flooding for negative secondary ions [1-3]. Our results show that both negative and positive ion yields obtained with $\mathrm{Ga}^{+}, \mathrm{He}^{+}$and $\mathrm{Ne}^{+}$bombardment may be increased by up to 4 orders of magnitude when using such reactive gas flooding (Figure 1). This optimization of secondary ion yields leads to detection limits varying from $10^{-3}$ to $10^{-6}$ for a lateral resolution between $10 \mathrm{~nm}$ and $100 \mathrm{~nm}$ (Figure 2).

The emitted secondary ions must be extracted by extraction optics with a maximized efficiency and without negatively impacting the focusing of the primary ion beam (broadening or distortion of the ion beam due to the electric fields). We have successfully designed such secondary ion extraction optics for several FIB-based instruments. The obtained extraction efficiency ranges from $40 \%$ (extraction voltages of a few $100 \mathrm{~V}$ ) up to $100 \%$ (extraction voltages of a few $\mathrm{kV}$ ).

These extraction optics are coupled to a specially designed compact high-performance magnetic sector double focusing mass spectrometer that we developed for the purpose of FIB-SIMS. The specifications of this mass spectrometer include highest transmission (100\%), high mass resolution $(\mathrm{M} / \Delta \mathrm{M}>2000)$, full mass range $(\mathrm{H}-\mathrm{U})$ and parallel detection of several masses. 
The results are very encouraging and the prospects of performing SIMS in combination with these highresolution imaging and nanofabrication instruments are very interesting. In addition, the combination of high-resolution microscopy and high-sensitivity chemical mapping on a single instrument represents a new level of correlative microscopy.

\section{References}

[1] P. Philipp et al., Int. J. Mass. Spectrom. 253 (2006) 71

[2] T. Wirtz et al., Appl. Phys. Lett. 101 (2012) 041601

[3] L. Pillatsch et al., Appl. Surf. Sei. 282 (2013) 908

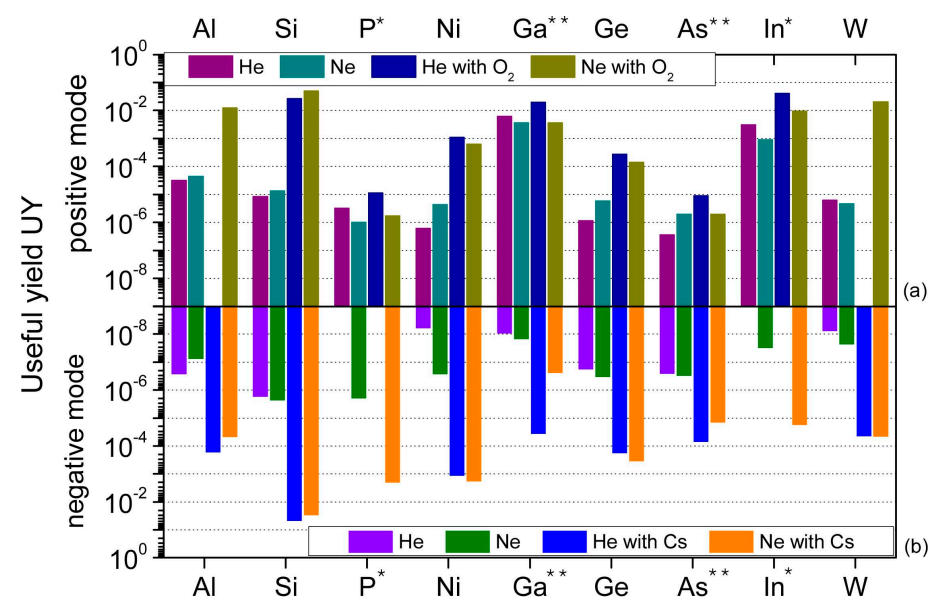

Matrix: ${ }^{*} \operatorname{InP} ;{ }^{*} \mathrm{GaAs}$

Analysed element

Figure 1: Enhancement of secondary ion yields using reactive gas flooding under $\mathrm{He}^{+}$and $\mathrm{Ne}^{+}$bombardment

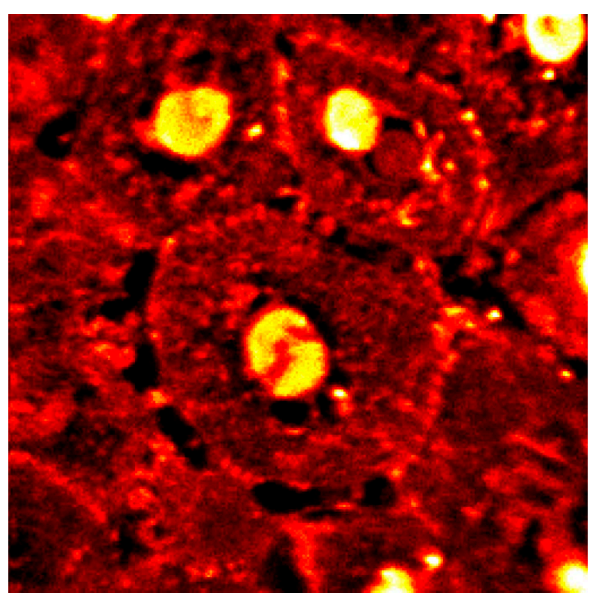

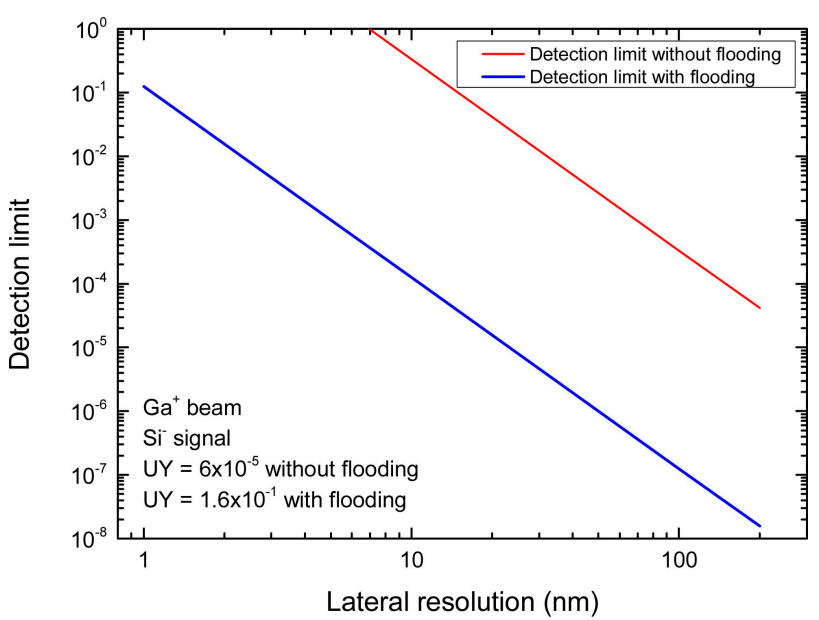

Figure 2: Detection limit using a $\mathrm{Ga}^{+} \mathrm{FIB}$ with and without $\mathrm{Cs}^{0}$ flooding vs. minimum feature size: example for the detection of $\mathrm{Si}^{-}$.

Figure 3: Ga+FIB-SIMS image of the Ca distribution in skin cells (field of view: $50 \times 50$ $\mu \mathrm{m}^{2}$ ) 